\title{
¿POR QUÉ UNA PALABRA SIGNIFICA LO QUE SIGNIFICA? COMENTARIOS A ESCEPTICISMO DEL SIGNIFICADO $Y$ TEORÍAS DE CONCEPTOS DE SÍLVIO MOTA PINTO
}

\author{
Axel Arturo Barceló Aspeitia \\ Instituto de Investigaciones Filosóficas \\ Universidad Nacional Autónoma de México \\ abarcelo@filosoficas.unam.mx
}

ReSumen: En esta nota critico la teoría de la interpretación de Mota Pinto (2009), arguyendo que predice significados donde intuitivamente no debería haberlos. De paso, critico su presentación de la perspectiva comunitarista del significado, distinguiendo entre dos tipos diferentes de hechos semánticos: el que una palabra signifique lo que significa en vez de significar otra cosa, y el que una expresión signifique lo que significa en vez de no significar nada.

PALABRAS CLAVE: contrastivismo, entendimiento, hechos, interpretación, Wittgenstein

SUMMARY: In this brief commentary, I argue that Mota Pinto's theory of interpretation (2009) predicts meanings where there are none. I also criticize his presentation of the communal perspective on meaning, drawing a distinction between two different kinds of semantic facts: a word meaning what it does rather than meaning something else, and such word meaning what it does rather than not meaning anything at all.

KEY WORDS: contrastivism, facts, interpretation, understanding, Wittgenstein

¿Qué determina que una palabra signifique lo que significa? Por un lado, al usar esa palabra, el hablante quiso decir algo en específico. Por el otro, quien lo escucha entiende lo que el hablante quiso decir porque sabe lo que esa palabra convencionalmente significa y sabe cómo usar esa información para interpretar las palabras que el hablante emitió en las circunstancias dadas. Intuitivamente, parece que, para que una palabra signifique lo que significa, es necesario que se satisfagan ambos tipos de condiciones: condiciones internas del hablante (como que tenga la intención de usar la palabra con un significado dado) y condiciones externas (como que el escucha conozca las convenciones de uso del lenguaje que se habla en dicha comunidad). Sin embargo, ¿qué sucede cuando no se satisfacen las condiciones de alguno de estos dos tipos? ¿Pierde la palabra su significado? ¿O basta que se satisfaga uno de los dos tipos de condiciones? Y, de ser así, ¿cuál de ellos? ¿Tiene prioridad algún tipo de condiciones sobre el otro? Ofrecer una respuesta novel a éstas y otras preguntas similares es el objetivo central del libro Escepticismo del significado y teorías de conceptos (2009) de Sílvio Mota Pinto. 
Según nos cuenta Mota Pinto, Ludwig Wittgenstein (1953) puso este tipo de preguntas en el centro de la discusión filosófica hace más de medio siglo. Probablemente, su interés principal era criticar a aquellos que, como Tarski, Russell, Hilbert y otros, trataban de fundamentar la lógica y la matemática sobre una teoría formal de la interpretación. Sin embargo, como bien señala Mota Pinto, también es probable que Wittgenstein se haya dado cuenta pronto de que sus críticas a dicho proyecto fundacionalista se extendían más allá del lenguaje matemático. Las preguntas formuladas por Wittgenstein ocupan hoy un lugar central en el esclarecimiento de los fundamentos metafísicos del significado. ¿Hay hechos semánticos? Si los hay, ¿qué tipo de hechos son? ¿Qué tipo de hecho podría ser el que, por ejemplo, "mero" a veces signifique simple y a veces puro? ¿Un hecho psicológico sobre los hablantes (cada uno de ellos en particular)? ¿O un hecho social sobre las comunidades de hablantes? La verdad de tesis semánticas tan simples como que mi nombre es "Axel" o que "alberca" y "piscina" son sinónimos ¿es objetiva o no? ¿Depende de nuestras capacidades cognitivas o es independiente de ellas? En su ambicioso volumen, además de ofrecer su propia y original respuesta a estas preguntas, Mota Pinto se propone hacer una revisión crítica de la discusión abierta por Wittgenstein y sus intérpretes. Para ello, suscribe las críticas de Wittgenstein al internismo del significado, es decir, a la hipótesis de que son las condiciones internas del hablante las que determinan el significado de una palabra (en un uso específico). Dicho de otro modo, Mota Pinto está de acuerdo con Wittgenstein en que, si existen los hechos del significado, éstos han de ser externos al hablante y, por lo tanto, no le son accesibles a través de la introspección. No obstante, reconoce que aún queda por determinar si esos hechos del significado han de depender (a) del punto de vista de la comunidad, o (b) del punto de vista del intérprete o escucha. Después de considerar las relativas ventajas y desventajas de cada opción, Mota Pinto termina favoreciendo la segunda.

Antes de revisar la propuesta positiva de Mota Pinto, permítaseme hacer una pequeña observación sobre la alternativa antiindividualista, a la que Mota Pinto llama "el punto de vista de la comunidad" [PVC]. Para éste, los hechos del significado (es decir, los hechos que hacen verdaderos los enunciados sobre el significado de un enunciado, palabra o frase) son hechos sociales o, en palabras de Mota Pinto, "comunitarios". Según el PVC, cuando Sílvio me habla por teléfono y pide con "Axel", el hecho de que su uso de esta palabra refiera a quien escribe estas líneas no es otro sino el hecho de que 
dicho uso concuerda con el sancionado por la comunidad lingüística, es decir, que cualquier otro miembro de nuestra comunidad de hablantes usaría esa misma palabra de la misma manera en las mismas circunstancias (presumiblemente, cualquier otra persona que pida hablar conmigo por teléfono tiene justificación para suponer que su interlocutor pertenece a la misma comunidad de hablantesescuchas, etcétera).

Nótese que la tesis fundamental del PVC es una tesis de identidad metafísica. No es que haya hechos comunitarios que expliquen los semánticos, sino que los hechos semánticos son hechos comunitarios. No es que me llame Axel (hecho semántico) porque así me llaman (hecho comunitario), sino que, en realidad, ambos hechos son el mismo. En palabras de Crispin Wright:

La corrección (o incorrección, según sea el caso) de la clase de enunciados "La palabra ' $e$ ' de $S$ significa tal y cual" debería identificarse con la concordancia (o discrepancia) entre su uso de ' $e$ ' y el sancionado por la comunidad lingüística en condiciones similares. (Wright 1980, p. 219; citado en Mota Pinto 2009, p. 136.)

Esta tesis metafísica es particularmente interesante porque postula la identidad entre lo que parecen ser dos tipos de hechos radicalmente diferentes. El primero es un hecho semántico sobre el significado de una expresión, mientras que el segundo es un hecho social sobre su uso. De ahí que, si el PVC tiene razón, los hechos del significado puedan identificarse - por lo menos qua hechos comunitarios- sin la necesidad de hacer mención alguna del significado. Nótese que, en la cita de Wright, en el segundo lado de la identidad no se hace mención alguna del significado. Parece que el "tal y cual" que se supone que significa " $e$ " desaparece. En consecuencia, aunque dicha identidad pueda capturar el carácter significativo de una expresión, no sirve de mucho para identificar su significado.

Como bien señala Mota Pinto, pese a ser una cuestión primordialmente metafísica, una buena teoría de los hechos semánticos también debe satisfacer lo que él llama "la condición epistémica", es decir, debe dar pie a una explicación epistemológica de nuestro conocimiento semántico, tanto de primera como de tercera personas (o, por lo menos, ser consistente con ella). En palabras de Mota Pinto: "La evidencia asociada al hecho constitutivo de que $S$ entiende '+' como significando suma debe ser capaz de justificar la creencia de que tales y cuales son las normas de uso para la palabra ' + ' del idiolecto de $S^{\prime \prime}$ (Mota Pinto 2009, p. 33). 
Por desgracia, el PVC no satisface esta condición epistémica. La evidencia asociada al hecho de que el uso que $S$ hace de "+" concuerda con el de su comunidad lingüística no es suficiente para justificar la creencia (ni de $S$, ni de nadie más) de que "+" efectivamente significa suma en el idiolecto de $S$, ya que es posible saber que la concordancia se da sin saber cuál es el significado de "+".

Para ilustrar este problema supongamos que alguien, $S$ (miembro de una comunidad lingüística distinta a la mía), usa una expresión que yo desconozco, por ejemplo, "Vijampaó". Supongamos, además, que de alguna manera me entero de que la comunidad a la que $S$ pertenece usaría "Vijampaó" exactamente como la usa $S$. No sé de qué uso se trata, pero sé que es el mismo que sanciona su comunidad. Según el PVC, conocer ese hecho comunitario es idéntico a conocer el hecho de que "Vijampaó" significa lo que sea que efectivamente signifique, pues ambos no son sino el mismo hecho. Sin embargo, está claro que, aun a sabiendas de que el uso de $S$ de "Vijampaó" es el que sanciona su comunidad, no sé qué significa dicha palabra. Es posible que haya acuerdo entre $S$ y su comunidad en el uso de "Vijampaó" y que, aun así, tal vocablo significara otra cosa.

La situación no cambia mucho si yo pertenezco a la misma comunidad lingüística que $S$. Aunque sea posible que yo simplemente proyecte mi uso sobre el de $S$ y reconozca así la concordancia, el tipo de conocimiento que obtengo de esta manera sigue siendo tan pobre como el que se obtiene desde fuera de la comunidad. De saber que $S$ usa la expresión como la usamos nosotros, sólo puedo inferir que el significado de "Vijampaó" en el uso de $S$ es el mismo que tendría en el uso que nosotros le damos. No sabré qué significado tiene "Vijampaó" en boca de $S$ si no sé antes qué significado tendría en mi boca, es decir, si no sé ya antes qué significado le damos a "Vijampaó" en mi comunidad. Sin embargo, el PVC no me dice cómo puedo saber esto (Recordemos, además, que ya hemos excluido la posibilidad de apelar a la introspección para saber qué significado tiene dicha expresión en boca nuestra) (Mota Pinto 2009, p. 162). Así, la concordancia de uso me puede decir que $S$ está usando "Vijampaó" de manera significativa, pero no me dice qué significa.

El problema fundamental que subyace en la discusión de Mota Pinto alrededor del PVC es una ambigüedad no reconocida en la expresión "el que una palabra signifique lo que significa". La misma expresión esconde dos hechos distintos: el que una palabra signifique lo que significa en vez de significar otra cosa, y el que una expresión signifique lo que significa en vez de no significar nada. 
Por ejemplo, no debe confundirse el hecho de que "perro" signifique lo que significa (es decir, una especie canina), en vez de significar otra cosa (un tipo de instrumento musical, un hueso del oído, etc.), con el hecho de que "perro" significa lo que significa, en vez de ser una frase sin sentido, un mero ruido o garabato. Me parece que, aun cuando el PVC pueda dar cuenta del segundo hecho, no logra captar el primero. El que la comunidad sancione el uso de un término tal vez sea suficiente para que éste sea significativo, es decir, para que signifique algo (para que signifique lo que significa en vez de no significar nada). Sin embargo, no parece suficiente para determinar su significado particular. Por lo tanto, no se puede identificar con el hecho de que ese uso de esa expresión signifique lo que significa en vez de significar otra cosa.

Como consecuencia de esta confusión, la teoría del PVC solamente está disponible para usos que efectivamente concuerdan con el de la comunidad. Si el uso que hace $S$ de una expresión " $e$ " no concuerda con el que harían los miembros de su comunidad lingüística, entonces no hay hecho de concordancia comunitario y por lo tanto tampoco hay hecho semántico alguno. En tal uso, "e" no significa nada. En consecuencia, es imposible que nadie use una expresión con un significado distinto al significado sancionado por la comunidad. Esto, porque los únicos usos significativos de las expresiones son aquellos que comparten los miembros de la comunidad. En otras palabras, los únicos significados que existen son los que concuerdan con el uso sancionado por la comunidad. Sin embargo, en la página 155, tras citar a Kripke (1982, p. 91), Mota Pinto escribe:

Puede ser que la discrepancia [en el uso de una expresión "+"] de Jones con Smith [donde Smith es un miembro de nuestra comunidad lingüística y Jones es la persona de cuyas palabras Smith quiere descubrir los significados que atribuye a sus palabras], sea lo suficientemente sistemática como para apoyar una hipótesis alternativa [a la de que usa "+" para la operación de adición, como lo haría cualquiera de nosotros]. Podríamos imaginar, por ejemplo, una situación en la que la mejor hipótesis que explique el comportamiento de Jones con respecto a "+" sea que usa este signo cuando quiere decir cuadición. (Mota Pinto 2009, p. 155)

Pero, según el PVC, es imposible que Jones (o quien sea) quiera decir cuadición con "+", si no hay una comunidad lingüística que sancione dicho uso. O "+" significa suma o no significa nada. Un signo, o bien significa lo que significa para la comunidad (o las comunidades) o bien no significa nada. 
He aquí una discrepancia importante entre el PVC y una teoría de la interpretación [TI] a la Quine-Davidson-Wittgenstein como la que Mota Pinto defiende. Para interpretar las emisiones de un hablante según el PVC, no basta observar el uso que dicho hablante da a sus expresiones, sino que es fundamental conocer primero el uso que les dan los miembros de su comunidad lingüística (y de alguna manera, como ya lo he señalado, saber también qué significado emana de dicho uso comunitario) y luego ver si el uso de este hablante particular concuerda con él o no. De haber concordancia, su uso significa lo que significa; si no la hay, no significa nada (Kripke 1982, p. 95; Goldfarb 1985, pp. 480-484).

Ahora bien, conforme a TI, es posible saber - por lo menos en principio - lo que un hablante quiere decir con una expresión sin conocer nada del uso que le dan a la misma expresión los otros miembros de su comunidad (es más, como Sílvio mismo señala al final del capítulo 5 (p. 211), ni siquiera es necesario que exista tal comunidad). Por lo tanto, uno puede reconocer significados discordantes, como la cuadición, inaceptables para el PVC. Lo único que es necesario para que alguien $S$ quiera decir $m$ por " $e$ " es usar " $e$ " de tal manera que un intérprete adecuado pueda abducir empíricamente, de dicho uso, que ése es el significado que $S$ le quiere dar. En otras palabras, el significado de " $e$ " por $S$ es $m$ si y sólo si adscribirle tal significado es la mejor hipótesis que empíricamente podría abducir una tercera persona $I$ de por qué $S$ usa " $e$ " como lo hace (Mota Pinto 2009, p. 190; Carston 2009).

Así, TI resuelve uno de los problemas fundamentales del PVC: explicar por qué, aun sin que haya convergencia en el uso de una expresión dentro de una comunidad, puede haber significado. Se puede decir, por lo tanto, que en TI el significado es más fácil de obtener que en el PVC. El problema, empero, es que el significado termina siendo demasiado sencillo de obtener. TI parece predecir significados donde intuitivamente no debería haberlos. Regresemos al ejemplo de "+". No importa cuán aparentemente aleatorias sean las respuestas que Jones dé a ecuaciones con este símbolo, siempre habrá una función matemática que codifique dichos usos (especialmente si se permite incorporar dependencias del contexto) y, en consecuencia, sería una buena hipótesis para explicar el uso que Jones da al mismo. Según TI, por lo tanto, dicha función sería el significado de "+" para Jones. No obstante, es fácil imaginar una situación en la cual Jones efectivamente responda al azar y, por lo tanto, no tenga en la mente ninguna función a computar en cada ecuación. Desde la tercera persona, y según los principios de TI, Smith podría obtener 
una función que correspondiera al uso de Jones para la cual éste no tendría ningún acceso privilegiado de primera persona. Dado que Mota Pinto exige, de toda teoría adecuada de los hechos semánticos, que respete este privilegio de la primera persona, TI resulta inaceptable conforme a sus propios criterios.

Mota Pinto trata de dar respuesta a este tipo de contraejemplos apelando a la intencionalidad, como condición necesaria para el significado (p. 191). Según su propia teoría de la interpretación, para que Jones pueda querer decir algo con su uso de "+", debe querer decir algo con su uso de "+". Pero esta intención, por supuesto, no debe ser privada, por las razones aducidas en el primer capítulo del libro (es decir, no puede ser un hecho interno). Por lo tanto, para que TI sea viable como teoría del significado, dicha intención debe ser también algo interpretable. ${ }^{2}$ Por ello y siguiendo a Wittgenstein, Mota Pinto sostiene que la conducta del hablante debe ser corregible, es decir, debe presentar, por ejemplo, "la conducta característica de corregir un lapsus linguae" (Wittgenstein 1953, § 54, apud Mota Pinto 2009, p. 187). Lamentablemente, esta respuesta lleva consigo sus propios problemas, los cuales la hacen inaceptable. No todo uso de toda expresión es explícita y manifiestamente intencional. Piensen ustedes en los textos escritos. Al leerlos, no tenemos acceso a las correcciones lingüísticas necesarias para tener evidencia de que el autor efectivamente quería decir algo con ellos. Nuestra hipótesis por defecto es que, cuando la gente habla o escribe, tiene la intención de usar sus expresiones con sentido. Pero rara vez tenemos evidencia empírica de ello. Por lo tanto, si dicha intención es esencial para saber que efectivamente alguien ha dicho algo significativo (lo cual parece ser así si queremos mantener la autoridad de la primera persona), y esa intención no es accesible empíricamente a través de la interpretación, entonces TI parece tener problemas graves para explicar cómo usamos el lenguaje a fin de comunicarnos.

Para TI, la única evidencia relevante sobre la cual podemos justificar nuestros juicios semánticos es la evidencia empírica, disponible desde la perspectiva de la tercera persona, sobre el uso de expresiones lingüísticas. Por desgracia, esta evidencia es insuficiente para justificar dichos juicios semánticos. En particular, es insuficiente para detectar (en todos los casos) que el uso que le da un hablante a una

${ }^{1}$ Pero no es suficiente que Jones tenga la intención de usar "+" significativamente, sino que es necesario que tenga la intención de usarla con un significado específico sobre el cual pueda construir pensamientos de segundo orden.

${ }^{2} \mathrm{O}$ debe ser un hecho comunitario, para que el PVC sea viable. 
expresión se acompaña de una intención comunicativa. Sin esta información, es imposible justificar nuestros juicios sobre cuáles usos son significativos y cuáles no. En otras palabras, así como la perspectiva de la comunidad tenía problemas insalvables tratando de explicar nuestro conocimiento de que una palabra significa lo que significa en vez de significar otra cosa, la teoría de la interpretación de Mota Pinto tiene problemas para explicar cómo sabemos que una palabra significa lo que significa en vez de no significar nada. ${ }^{3}$

\section{BIBLIOGRAFÍA}

Carston, R., 2009, "Relevance Theory: Contextualism or Pragmaticism", Working Papers in Linguistics, vol. 21, pp. 19-26.

Goldfarb, W., 1985, "Kripke on Wittgenstein on Rules", The Journal of Philosophy, vol. 82, pp. 471-488.

Kripke, S., 1982, Wittgenstein on Rules and Private Language, Harvard University Press, Cambridge, Mass. [Versión en castellano: Wittgenstein a propósito de reglas y lenguaje privado: una exposición elemental, trad. Jorge Rodríguez, Tecnos, Madrid, 2006.]

Mota Pinto, S., 2009, Escepticismo del significado y teorías de conceptos, trad. Laura E. Manríquez, Anthropos/Universidad Autónoma Metropolitana-Iztapalapa, Barcelona/México.

Wittgenstein, L., 1953, Philosophical Investigations, Blackwell, Oxford. [Versión en castellano: Investigaciones filosóficas, trad. Adolfo García Suárez y Ulises Moulines, Instituto de Investigaciones Filosóficas-UNAM, México, 1988.]

Wright, C., 1980, Wittgenstein on the Foundations of Mathematics, Harvard University Press, Cambridge, Mass.

Recibido el 2 de enero de 2010; revisado el 20 de agosto de 2010; aceptado el 8 de septiembre de 2010.

${ }^{3}$ Agradezco a Sílvio Mota Pinto el haberme invitado a comentar su libro, y sus alentadores comentarios a una versión previa de este texto. Agradezco también las sugerencias y señalamientos de los dictaminadores de Crítica y el apoyo del proyecto CONACYT 105949 "Los problemas del conocimiento y la comprensión en matemáticas". 